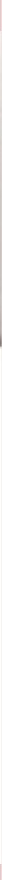

\title{
9. VDI-Tagung
}

Schwingungen von

Windenergieanlagen 2018

\author{
Bremen, 12. und 13. Juni 2018
}




\section{VDI-BERICHTE \\ Herausgeber: \\ VDI Wissensforum GmbH}


Bibliographische Information der Deutschen Nationalbibliothek

Die Deutsche Nationalbibliothek verzeichnet diese Publikation in der Deutschen Nationalbibliographie;

detaillierte bibliographische Daten sind im Internet unter www.dnb.de abrufbar.

Bibliographic information published by the Deutsche Nationalbibliothek (German National Library)

The Deutsche Nationalbibliothek lists this publication in the Deutsche Nationalbibliographie (German National Bibliography); detailed bibliographic data is available via Internet at www.dnb.de.

\section{(c) VDI Verlag GmbH · Düsseldorf 2018}

Alle Rechte vorbehalten, auch das des Nachdruckes, der Wiedergabe (Photokopie, Mikrokopie), der Speicherung in Datenverarbeitungsanlagen und der Übersetzung, auszugsweise oder vollständig.

Der VDI-Bericht, der die Vorträge der Tagung enthält, erscheint als nichtredigierter Manuskriptdruck.

Die einzelnen Beiträge geben die auf persönlichen Erkenntnissen beruhenden Ansichten und Erfahrungen der jeweiligen Vortragenden bzw. Autoren wieder. Printed in Germany. 


\section{Inhalt}

\section{BIG DATA Anwendungen - Predictive Maintenance, Betriebsoptimierung und Weiterbetrieb}

Condition Monitoring Systeme als Datenlieferant für Windenergieanlagen. . . . . . . . . . 1

J. Folmer, E. Becker, PRÜFTECHNIK Condition Monitoring GmbH, Ismaning

Predictive Analytics als Entscheidungsgrundlage $\ldots \ldots \ldots \ldots \ldots \ldots \ldots \ldots$

F. Wagner, STEAG Energy Services GmbH, Essen

Einfluss nicht planmäßiger Schwingungen auf die Weiterbetriebsdauer von

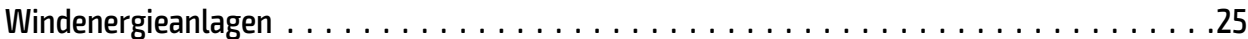

A. Andreä, Axel Andreä Consulting and Engineering, Köln;

U. Moritz, windexperts Prüfgesellschaft $\mathrm{mbH}$,

c/o energie profile Ingenieurbüro Glocker, Memmingen

\section{Schallmessung und Schallminderung}

Einsatz von Körperschalltilgern zur Reduzierung von Tonalitäten von Windenergieanlagen . . .39

F.-D. Krull, L. Schneider,

ESM Energie- und Schwingungstechnik Mitsch GmbH, Heppenheim

\section{Signalanalyse und Simulation}

Nutzung von Inertialmesstechnik zur Stützung modellbasierter Berechnungsalgorithmen

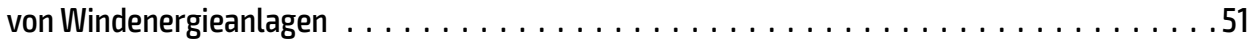

T. Meyer, A. Bartschat, F. Meng, A. Zuga, Fraunhofer IWES, Bremerhaven;

T. Martin, M. Krings, NG LITEF, Freiburg

Operative Modalanalyse an Windenergieanlagen der 2 MW und 3 MW-Klasse . . . . . . . . . .63

J. Zierath, R. Bockhahn, S.-E. Rosenow, WZE Wind to Energy GmbH, Rostock;

R. Rachholz, A. Schulze, J. Luthe, C. Woernle, Universität Rostock, Rostock 
Multidisziplinäre Simulation einer WEA zur Analyse der Kopplungseffekte von

Strukturdynamik und netzstützenden Regelungsverfahren . . . . . . . . . . . . . . . .77

B. Fischer, P. Brosche, Fraunhofer IEE, Abteilung Regelungstechnik, Kassel;

A. Rezaeian, M. Sayed, MesH Engineering GmbH, Stuttgart

Experimentelle Modalanalyse an einem aeroelastisch optimierten Rotorblatt mit

Biege-Torsions-Kopplung im Projekt SmartBlades2 . . . . . . . . . . . . . . . . . . . . . .91

J. Gundlac, Y. Govers, Institut für Aeroelastik, Deutsches Zentrum für

Luft- und Raumfahrt (DLR), Göttingen

\section{Condition Monitoring - Felderfahrung}

Einfache Erfassung und Bewertung der Rotorunwucht - Ein Vergleich der Kennwerte nach VDI 3834-1 mit testgewichts- und modellbasierten Verfahren . . . . . . . . . . . . . . . . 105

U. Oertel, H. Fritsch, Bachmann Monitoring GmbH , Rudolstadt;

F. Krause, BKW Erneuerbare Energien GmbH, Berlin

Smart Condition Monitoring am Triebstrang von WEA's . . . . . . . . . . . . . . . . . . 115

R. Wirth, GfM Gesellschaft für Maschinendiagnose mbH, Berlin

\section{Structural Health Monitoring - Entwicklungsfelder} und Anwendung

Rotorblattsensorik in über 200 Anlagen weltweit Felddaten und wertvolle

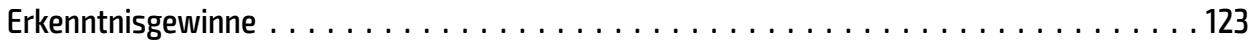

B. Kuhnle, fos 4X GmbH, München 


\section{- Tagungsleiter}

Dipl.-Ing. Thomas Gellermann, Leitender Oberingenieur/Gutachter, Allianz Zentrum für Technik, Allianz Risk Consulting GmbH, München

\section{- Programmausschuss}

Dr. rer. nat. Edwin Becker, Leiter Service \& Diagnose Center, Prüftechnik Condition Monitoring GmbH, Ismaning

Dr. Walter Holweger, Senior Key Expert Grundlagen Tribologie, Schaeffler Technologies AG \& Co. KG, Herzogenaurach

Dr.-Ing. Martin Huhn, Leiter der Abteilung Turbine \& Load Simulation, Suzlon Energy GmbH, Rostock

Dr.-Ing. Klaus Kaiser, Senior Engineer Technical Lead, ADWEN GmbH, Hamburg

Dr.-Ing. Martin Klönne, Head of Drivetrain/DE, Drive Train R\&D, Vestas Nacelles Deutschland GmbH, Dortmund

Martin von Mutius, Head of Analysis \& Certification, Product Development, Senvion SE, Osterrönfeld

Dr. Samer Mtauweg, Innovation Technology Expert, I4E Innovation for ENERCON GmbH, Bremen Dr.-Ing. Karl Steingröver, Sachverständiger, Erneuerbare Energien Zertifizierung, Germanischer Lloyd Industrial Services GmbH, Hamburg

Prof. Dr.-Ing. Horst Wölfel, Inhaber, Wölfel Beratende Ingenieure GmbH + Co. KG, Höchberg Dipl.-Chem. Mark Zundel, Head of Engineering, Siemens AG, Voerde 


\section{- Fachlicher Träger}

\section{Fachgesellschaft VDI-GPP}

Die VDI-Gesellschaft Produkt- und Prozessgestaltung bietet mit ihren Fachbereichen für alle Branchen abgesichertes Wissen zur Gestaltung von Produkten und Prozessen sowie deren Optimierung bezüglich Qualität, Zeit und Kosten-Nutzenverhältnis.

www.vdi.de/gpp

\section{Fachbereich Schwingungstechnik}

Der Fachbereich Schwingungstechnik befasst sich unter anderem mit der Messung von Schwingungen und der Bewertung der Ergebnisse. Es werden Methoden entwickelt, um Schwingungen zu analysieren und durch aktive und passive Schwingungsminderung zu beeinflussen. Handlungsempfehlungen in Form von VDI Richtlinien, Erfahrungsaustausch und Veranstaltungen sind Ergebnisse der Aktivitäten.

www.vdi.de/schwingungstechnik 